\title{
Exploration of predictors of benefit from nivolumab monotherapy for patients with pretreated advanced gastric and gastroesophageal junction cancer: post hoc subanalysis from the ATTRACTION-2 study
}

\author{
Yoon-Koo Kang ${ }^{1}$ D . Satoshi Morita ${ }^{2} \cdot$ Taroh Satoh $^{3} \cdot$ Min-Hee Ryu ${ }^{1} \cdot$ Yee Chao ${ }^{4} \cdot$ Ken Kato ${ }^{5} \cdot$ Hyun Cheol Chung $^{6}$. \\ Jen-Shi Chen ${ }^{7}$. Kei Muro ${ }^{8}$. Won Ki Kang ${ }^{9} \cdot$ Kun-Huei Yeh ${ }^{10}$. Takaki Yoshikawa ${ }^{11,26}$. Sang Cheul Oh ${ }^{12} \cdot$ Li-Yuan Bai $^{13}$. \\ Takao Tamura ${ }^{14,27}$. Keun-Wook Lee ${ }^{15}$. Yasuo Hamamoto ${ }^{16}$. Jong Gwang Kim ${ }^{17}$. Keisho Chin ${ }^{18}$. Do-Youn Oh ${ }^{19}$. \\ Keiko Minashi ${ }^{20}$. Jae Yong Cho ${ }^{21} \cdot$ Masahiro Tsuda ${ }^{22} \cdot$ Hiroki Sameshima $^{23} \cdot$ Li-Tzong Chen $^{24,25} \cdot$ Narikazu Boku $^{5}$
}

Received: 28 April 2021 / Accepted: 4 August 2021 / Published online: 4 September 2021

(C) The Author(s) 2021

\begin{abstract}
Background The phase 3 ATTRACTION-2 study demonstrated that nivolumab monotherapy was superior to placebo for patients with pretreated advanced gastric or gastroesophageal junction cancer, but early progression of tumors in some patients was of concern.

Methods This post hoc analysis statistically explored the baseline characteristics of the ATTRACTION-2 patients and extracted a single-factor and double-factor combinations associated with early disease progression or early death. In the extracted patient subgroups, the 3-year restricted mean survival times of progression-free survival and overall survival were compared between the nivolumab and placebo arms.

Results Two single factors (age and peritoneal metastasis) were extracted as independent predictors of early progression, but none of them, as a single factor, stratified patients into two subgroups with significant differences in restricted mean survival time. In contrast, two double-factor combinations (serum sodium level and white blood cell count; serum sodium level and neutrophil-lymphocyte ratio) stratifying patients into two subgroups with significant differences in the restricted mean survival time were extracted. Additional exploratory analysis of a triple-factor combination showed that patients aged $<60$ years with peritoneal metastasis and low serum sodium levels (approximately $7 \%$ of all patients) might receive less benefit from nivolumab, and patients aged $\geq 60$ years with no peritoneal metastasis and normal serum sodium levels might receive higher benefit.

Conclusions A combination of age, peritoneal metastasis, and serum sodium level might predict benefit from nivolumab as salvage therapy in advanced gastric or gastroesophageal junction cancer patients, especially less benefit for patients having all three risk factors.
\end{abstract}

Keywords Gastric cancer $\cdot$ Gastroesophageal junction $\cdot$ Nivolumab $\cdot$ Early progression $\cdot$ Benefit predictors

\section{Background}

Immune checkpoint inhibitors (ICIs) have led to substantial breakthroughs in cancer therapy [1]. By blocking an immune checkpoint pathway, ICIs promote the activation of cytotoxic $\mathrm{T}$ lymphocytes, thereby facilitating the removal of tumor cells. ICIs have been approved for therapy of multiple

Yoon-Koo Kang

ykkang@amc.seoul.kr

Extended author information available on the last page of the article cancers. A remarkable advantage of ICI therapy is its longterm survival benefit. However, it has become evident that some patients respond poorly to ICI therapy, and immunerelated adverse events (irAEs) are of great concern, particularly for non-responders [2-4]. Treatment effect predictors are useful in the decision-making in daily practice of clinicians for optimizing patient benefit [5-7].

Monoclonal antibodies against human programmed-death receptor 1 (PD-1), such as nivolumab and pembrolizumab, inhibit a PD-1-mediated suppression signal of cytotoxic $T$ lymphocytes [8]. Nivolumab and pembrolizumab have been proven to be effective for several types of cancer, including 
malignant melanoma, non-small cell lung carcinoma, and gastric cancer. The most frequently evaluated effect predictor for these PD-1 blockades is the programmed-death ligand 1 (PD-L1) expression level because PD-L1 binds to PD-1 on T lymphocytes to induce the PD-1-mediated suppression signal $[6,9]$. Indeed, several approved indications are linked to testing PD-L1 expression on tumor cells and tumor-infiltrating immune cells [7]. On the other hand, some clinical trials have reported a lower correlation between the efficacy of PD-1 blockades and PD-L1 expression. Thus, it remains a controversial issue whether PD-L1 expression is a widely applicable predictor of benefit from PD-1 blockades [7]. Likewise, other potential biomarkers, such as the tumor mutation burden, microsatellite instability, and $\mathrm{CD} 8^{+} \mathrm{T}$ lymphocytes, have been evaluated, and some of them are linked to approved indications $[10,11]$, but no powerful predictors of PD-1 blockade efficacy have been discovered [12].

The phase 3 ATTRACTION-2 study evaluated the efficacy and safety of nivolumab monotherapy as third-line or later-line treatment in advanced gastric or gastroesophageal junction (G/GEJ) cancer patients [13]. In ATTRACTION-2, nivolumab significantly reduced risks of death and disease progression compared with placebo [13]. A subsequent follow-up analysis showed a better 3-year overall survival (OS) rate in the nivolumab arm [14]. Although nivolumab apparently provides benefit to G/GEJ cancer patients, early disease progression within two months was observed in approximately half of both the nivolumab- and placebotreated patients, suggesting that some patients benefited poorly from nivolumab. In ATTRACTION-2, PD-L1 expression by tumor cells was not predictive of treatment efficacy, and other potential biomarkers could not be evaluated due to data limitations [13].

To help clinician's decision-making, this post hoc subanalysis in ATTRACTION-2 aimed to explore the baseline characteristics of patients that were associated with low and high benefit from nivolumab as salvage therapy for gastric cancer. Conventional univariable and multivariable analyses have been often used to identify predictive biomarkers. However, since these conventional analyses consider an outcome of only a single treatment arm, prognostic but not predictive factors may be extracted. Furthermore, combinations of two or more factors are rarely analyzed. Recently, several statistical methods have been developed to overcome these limitations [15], one of which is the "BaPoFi" method [16]. To explore subgroups that are most likely to benefit from one of two treatment arms in a randomized trial, the BaPoFi method can evaluate the difference in treatment effects between two treatment arms in all possible subgroups classified by a combination of multiple factors as well as by a single factor. In this study, $\mathrm{BaPoFi}$ analysis, as well as conventional univariable and multivariable analyses, was performed to extract predictive factors.
The hazard ratio is a well-established evaluation statistic, but the underlying proportional hazards assumption may be violated in ICI therapy [17]. Therefore, the restricted mean survival time (RMST) has been proposed as an alternative and statistically valid method for assessing the effectiveness of ICI therapy $[18,19]$. To evaluate the treatment effects of subgroups in this post hoc subanalysis, we compared the estimated RMST of the progression-free survival (PFS) and that of the OS between the nivolumab and placebo arms.

\section{Methods}

\section{Study design}

ATTRACTION-2 was a randomized, double-blind, placebocontrolled, multicenter phase 3 clinical trial conducted in Japan, South Korea, and Taiwan, and was registered with ClinicalTrials.gov, number NCT02267343. The detailed design of ATTRACTION-2 was described previously [13]. Additional informed consent specific for this post hoc study was not obtained from the ATTRACTION-2 participants.

\section{Patients}

A total of 493 metastatic G/GEJ cancer patients were enrolled in ATTRACTION-2, 330 and 163 of whom were randomly allocated to receive nivolumab and placebo, respectively. Two patients in the placebo arm were excluded from this post hoc subanalysis due to the study drugs not being administered to them. Because patient data, such as body mass index and C-reactive protein levels, were missing for some patients, the $\mathrm{BaPoFi}$ and RMST analyses included 318 and 155 patients in the nivolumab and placebo arms, respectively, all of whom have all required data (Online Resource Fig. S1a).

\section{Variables}

In this post hoc subanalysis, we included patients with OS and PFS data of a minimum 3-year follow-up [14]. Baseline characteristics, clinical history, and clinical laboratory values of the patients were used in the statistical analyses (Online Resource Table S1). These data were recorded at enrollment to ATTRACTION-2, except for primary tumor site and histological classification that were obtained at diagnosis of G/GEJ cancer. The original data for metastasis were those collected at diagnosis of G/GEJ cancer, and therefore, we generated data on metastasis using computed tomography or magnetic resonance imaging records regarding target and non-target legions at enrollment to the study. The cutoff value for each laboratory factor was institutional lower or 
upper limits, medians, tertiles, or clinically meaningful values (Online Resource Table S1).

\section{Statistical methods for extraction}

To extract independent factors associated with disease progression, we used two models for conventional univariable and multivariable analyses. Logistic regression analyses assessed PFS within 8 weeks in the nivolumab arm, when the Kaplan-Meier curves of PFS began to markedly separate between the nivolumab and placebo arms. We also assessed PFS over time by Cox regression analyses. The multivariable analysis assessed factors that was identified with $p$ values less than 0.05 in the preceding univariable analysis. Then, we focused on the independent factors related with these two outcomes of PFS that were extracted from both multivariable logistic and Cox analyses with $p$ values less than 0.05 . Here we used PFS data but not OS data because we focused on early progression rather than OS.

To consider double-factor combinations, we conducted the BaPoFi method. The BaPoFi method considers the problem to identify effect predictors using Bayesian additive regression trees (BART) and evaluate subgroups with differential effects as a Bayesian decision problem. In the BART computation, we assume a conjugate normal prior for the mean parameters of the terminal nodes and assume a conjugate inverse-chi-squared prior for the variance parameter. In this study, the BaPoFi analysis considered the PFS rates at 8 weeks and OS rates at 5.26 months between the nivolumab and placebo arms as independent binary definitions: survival and non-survival. The OS rates of 5.26 months was the median OS in the nivolumab arm of the total patient population. Expected utility with respect to future outcomes were evaluated for each single factor and for a combination of double factors. Single factors and combinations of double factors ranked at the top five of preferable outcomes were extracted. Of note, the BaPoFi method focuses on a meaningful difference between the two treatment arms, thereby potentially avoiding extraction of mere prognostic factors even if OS rates are used.

\section{Statistical methods for validation}

A total of 318 patients in the nivolumab arm and 155 patients in the placebo arm were classified into two categories based on the cutoff value of a single factor: a low-benefit group and high-benefit group (Online Resources Fig. S1a and $b$ ). A double-factor combination classified patients into three categories: a low-benefit group that included patients with two risk factors in the double-factor combination, a high-benefit group that included patients with no risk factors in the double-factor combination, and the others with either one risk factor (Online Resources Fig. S1a and b). To assess the benefit from nivolumab in each subgroup, Kaplan-Meier curves of OS and PFS were estimated between the nivolumab and placebo arms, followed by estimation of the RMST at 36 months after the first dose (Online Resources Fig. S1c). Here, we considered a difference of RMST, but not a ratio of RMST, as the effect measure because a treatment benefit may be intuitively understandable for clinicians and patients. We defined $\triangle$ RMST as the difference in the estimated RMST between the nivolumab and placebo arms (nivolumab minus placebo), which could be considered the potential benefit from nivolumab (Online Resources Fig. S1c). Also, we defined $\Delta \Delta$ RMST as the difference in the $\triangle$ RMST between the low- and high-benefit groups (high minus low), which would reflect the difference in benefit from nivolumab between them (Online Resources Fig. S1d). Thus, factors associated with a larger $\Delta \Delta$ RMST of both PFS and OS are better predictors of benefit from nivolumab. A $p$ value below 0.05 was considered to indicate statistical significance. Best overall responses and objective response rates in certain patient subgroups were calculated as described previously [13].

\section{Results}

\section{RMST analysis for the entire ATTRACTION-2 patient population}

Estimated RMSTs at 36 months of OS and PFS for the entire intention-to-treat population in the nivolumab arm $(N=330)$ were 9.6 and 4.7 months, respectively, and those in the placebo arm $(N=163)$ were 6.0 and 2.4 months, respectively. The $\triangle$ RMSTs of OS and PFS between the nivolumab and placebo arms were 3.6 months (95\% confidence interval [CI], 2.10-5.05; $p<0.0001$ ) and 2.3 months (95\% CI, 1.37-3.22; $p<0.0001$ ), respectively, indicating that the RMST analysis demonstrated significant improvement by nivolumab in both OS and PFS in the entire ATTRAC TION-2 patient population.

\section{Conventional univariable and multivariable analyses}

Conventional univariable logistic and Cox regression analyses individually identified around 20 factors associated with disease progression in the nivolumab arm (Online Resource Tables S2 and S3). Because multiple cutoff values were extracted for age (50,60, 65, and 70 years), we conducted Kaplan-Meier and RMST analyses of PFS for the patient subgroups classified according to these cutoff values. As a result, an age of 60 years, but not ages of 50,65 , or 70 years, classified patients into low- and high-benefit groups showing statistically significant differences in $\Delta \Delta$ RMST (Online 
Resource Table S4). Thus, conventional multivariable analyses were conducted using 60 years as the cutoff value for age. The multivariable logistic regression analysis identified age, peritoneal metastasis, and liver metastasis as risk factors of disease progression within 8 weeks and the multivariable Cox regression analysis identified age, lymph node metastasis, peritoneal metastasis, white blood cell count, and lactate dehydrogenase value as being independent factors associated with disease progression (Online Resource Tables S2 and S3). Age and peritoneal metastasis that were extracted from both multivariable analyses were used for subsequent analyses (Table 1).

The Kaplan-Meier and RMST analyses of OS and PFS for the patient subgroups classified by these independent factors indicated that the $\Delta \Delta$ RMST of PFS between the lowbenefit ( $<60$ years) and high-benefit ( $\geq 60$ years) groups classified by age was 1.9 months and was significantly different, whereas $\triangle \triangle \mathrm{RMST}$ of OS did not show a statistically significant difference between the two groups (Table 1; Online Resource Fig. S2). A significant difference in the $\Delta \Delta$ RMST between the low-benefit (with peritoneal metastasis) and high-benefit (without peritoneal metastasis) groups classified by peritoneal metastasis was observed for OS (4.2 months) but not for PFS. Thus, as a single factor, none of these independent factors showed statistically significant differences between the two groups in both $\Delta \Delta \mathrm{RMST}$ values of OS and PFS.

\section{BaPoFi analysis}

Besides the conventional multivariable analyses, we conducted an analysis using the $\mathrm{BaPoFi}$ method to identify double-factor combinations associated with preferable OS or PFS in the nivolumab arm compared with the placebo arm because the BaPoFi method has an advantage on exploring high-benefit groups rather than low-benefit groups. Four

Table 1 Independent factors identified in both conventional logistic and Cox regression multivariable analyses as being significantly associated with early progression in the nivolumab arm

\begin{tabular}{|c|c|c|}
\hline Factors [Cutoff] & $\begin{array}{l}\Delta \Delta \mathrm{RMST}^{\mathrm{a}} \text { of OS } \\
(95 \% \mathrm{CI}), \text { months }\end{array}$ & $\begin{array}{l}\Delta \Delta \mathrm{RMST}^{\mathrm{a}} \text { of PFS } \\
(95 \% \mathrm{CI}), \text { months }\end{array}$ \\
\hline Age $[<60$ vs. $\geq 60]$ & $\begin{array}{l}2.7(-0.09-5.47) \\
p=0.0584\end{array}$ & $\begin{array}{l}1.9(0.14-3.59) \\
p=0.0341\end{array}$ \\
\hline $\begin{array}{c}\text { Metastasis [Peritoneal } \\
\text { metastasis vs. No] }\end{array}$ & $\begin{array}{l}4.2(1.20-7.30) \\
p=0.0063\end{array}$ & $\begin{array}{l}0.9(-0.90-2.76) \\
p=0.3202\end{array}$ \\
\hline
\end{tabular}

Conventional multivariable analysis was conducted, which included the factors identified in the preceding univariable analysis as being significantly associated with early progression in the nivolumab arm

$C I$ confidence interval, $O S$ overall survival, $P F S$ progression-free survival, $R M S T$ restricted mean survival time

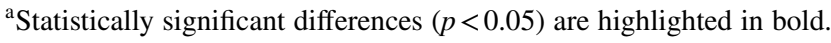
The definition of $\Delta \Delta$ RMST is described in "Methods" double-factor combinations were extracted in both OS and PFS analyses (Online Resource Table S5 shows the top five subgroups). We classified patients into three subgroups using the identified double-factor combinations: a highbenefit group with the two preferable factors (for example, normal serum sodium level and normal white blood cell count [WBC]), a low-benefit group with two risk factors (for example, lower serum sodium level and higher WBC), and others with one preferable and one risk factors (Online Resource Fig. S1b). The RMST analyses showed statistically significant differences in the $\Delta \Delta \mathrm{RMST}$ of both OS and PFS between the low- and high-benefit groups in two of the double-factor combinations: The $\Delta \Delta$ RMST values of OS and PFS between low- and high-benefit groups classified by the combination of serum sodium level (lower vs. normal range) and WBC (higher vs. normal range) were 2.7 and 1.9 months, respectively, and those classified by the combination of serum sodium level (lower vs. normal range) and neutrophil-lymphocyte ratio ([NLR]; highest tertile vs. middle or lowest tertile) were 2.6 and 2.0 months, respectively (Table 2; Figs. 1 and 2; Online Resource Fig. S3).

\section{Exploratory analyses}

The BaPoFi analysis-extracted combinations included four single factors (serum sodium level, serum chloride level, WBC, and NLR; all were laboratory data), which were totally different from the two independent factors extracted in the conventional multivariable analyses (age and peritoneal metastasis; these were patient's condition). However, the $\Delta \Delta$ RMST values of OS and PFS in patient subgroups classified by the BaPoFi analysis-extracted combinations were numerically smaller than those in the subgroup classified by the combination of age and peritoneal metastasis. To test the possibility that any double-factor combinations of these six extracted factors, 15 combinations in total, defined the low- and high-benefit groups with a larger $\Delta \Delta$ RMST, we conducted a further exploratory analysis for additional 11 possible double-factor combinations; the 4 combinations identified by the $\mathrm{BaPoFi}$ analysis were already analyzed above. Among them, seven combinations of double factors showed statistically significant differences in the $\Delta \Delta \mathrm{RMST}$ values of both OS and PFS between the low- and high-benefit groups (Tables 2,3 ). The largest $\Delta \Delta$ RMST of OS was 6.8 months, which was shown for the patient subgroups classified using an age of 60 years and serum sodium level. In addition, while the $\Delta \Delta$ RMST values of OS in the top three combinations (age and serum sodium level, age and peritoneal metastasis, and peritoneal metastasis and serum sodium level) were remarkably larger than those in other combinations, these constituents mutually overlapped.

Thus, in a further exploratory analysis, we evaluated a combination of the top three factors: age, peritoneal 
Table 2 Top four double-factor combinations identified in both the OS and PFS subanalyses in the BaPoFi analysis

\begin{tabular}{|c|c|c|c|}
\hline $\begin{array}{l}\text { Factors [classification], low- vs. high- } \\
\text { benefit groups }\end{array}$ & $\begin{array}{l}n \text { (nivolumab/ } \\
\text { placebo) }\end{array}$ & $\begin{array}{l}\Delta \Delta \mathrm{RMST}^{\mathrm{a}} \text { of OS }(95 \% \\
\mathrm{CI}), \text { months }\end{array}$ & $\begin{array}{l}\Delta \Delta \mathrm{RMST}^{\mathrm{a}} \text { of PFS } \\
(95 \% \mathrm{CI}), \text { months }\end{array}$ \\
\hline $\mathrm{Na}$ [Lower] + WBC [Higher] vs & $18 / 5$ vs & $2.7(0.55-4.95)$ & $1.9(0.52-3.31)$ \\
\hline $\mathrm{Na}$ [Normal] + WBC [Normal] & $222 / 114$ & $p=0.0143$ & $p=0.007$ \\
\hline $\mathrm{Na}$ [Lower] + NLR [High] vs & $30 / 17$ vs & $2.6(0.23-5.01)$ & $2.0(0.44-3.54)$ \\
\hline $\mathrm{Na}$ [Normal] + NLR [Middle/low] & $190 / 89$ & $p=\mathbf{0 . 0 3 1 9}$ & $p=0.0116$ \\
\hline $\mathrm{Na}$ [Lower] + CL [Lower] vs & $29 / 12$ vs & $1.3(-1.82-4.49)$ & $1.1(-0.62-2.75)$ \\
\hline $\mathrm{Na}[$ Normal $]+\mathrm{CL}[$ Normal $]$ & $246 / 123$ & $p=0.4077$ & $p=0.2141$ \\
\hline CL [Lower] + NLR [High] vs & $26 / 16$ vs & $1.8(-1.02-4.65)$ & $1.6(-0.03-3.30)$ \\
\hline CL [Normal] + NLR [Middle/low] & $199 / 96$ & $p=0.2102$ & $p=0.055$ \\
\hline
\end{tabular}

$C I$ confidence interval, $C L$ serum chloride level, $N a$ serum sodium level, $N L R$ neutrophil-lymphocyte ratio, $O S$ overall survival, $P F S$ progression-free survival, $R M S T$ restricted mean survival time, $W B C$ white blood cell count

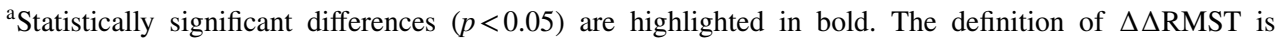
described in "Methods" a

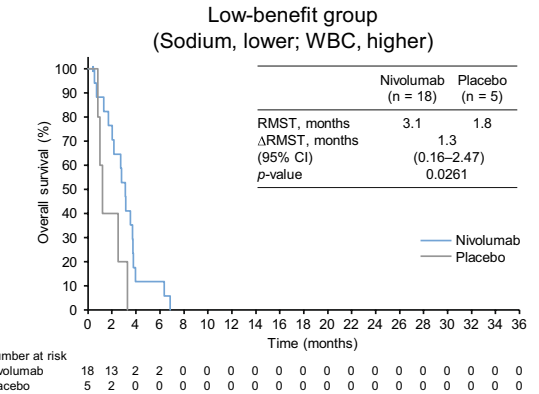

b

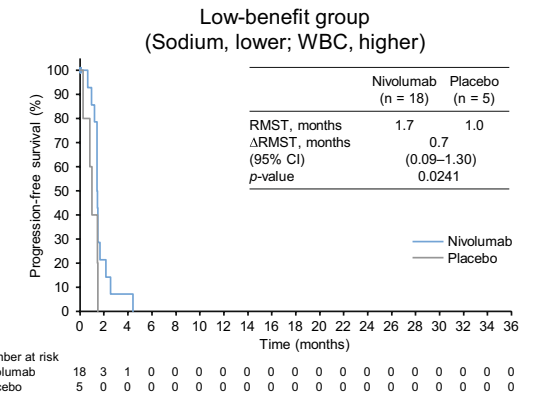

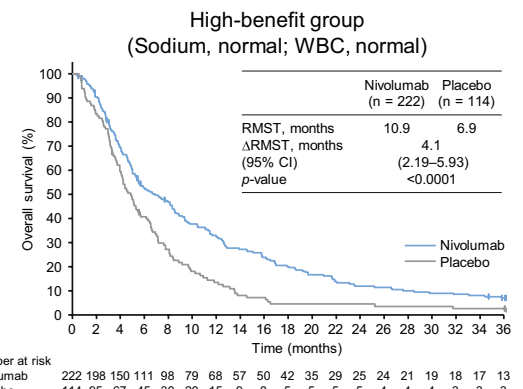

Number at risk
Nivolumab
Placebo

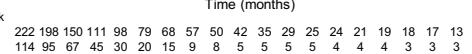

High-benefit group

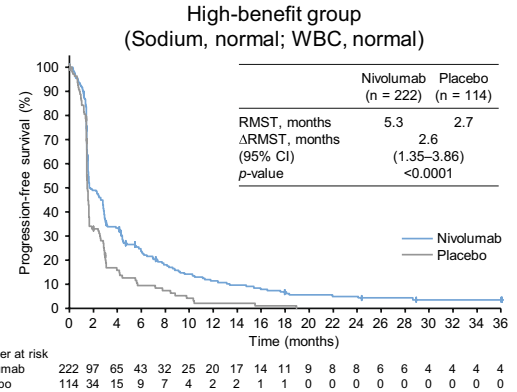

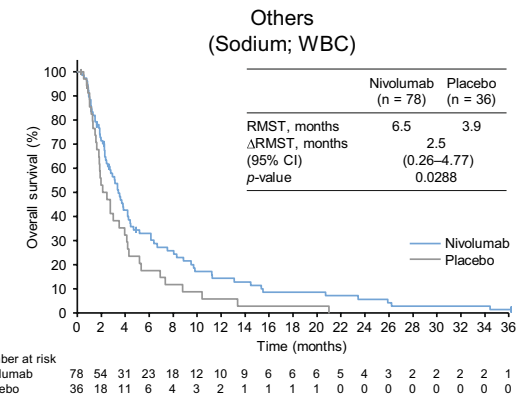

Others

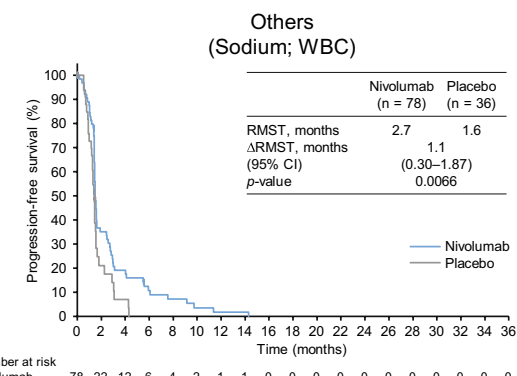

Fig. 1 Classification by the double-factor combination of serum sodium level and WBC. The Kaplan-Meier curves of the OS (a) and PFS (b) with the RMST values in the low-benefit group (left), in the high-benefit group (center), and in the others (right) are shown. CI confidence interval, $O S$ overall survival, $P F S$ progression-free survival, $R M S T$ restricted mean survival time, $\triangle R M S T$ difference in RMST between the nivolumab and placebo arms, WBC white blood cell count metastasis, and serum sodium level. Patients younger than 60 years with peritoneal metastasis and with a lower serum sodium level who were classified into the markedly lowbenefit group accounted for $6.3 \%$ and $7.7 \%$ of all patients in the nivolumab and placebo arms, respectively, and patients aged 60 years or older without peritoneum metastasis and with normal serum sodium level who were classified into the markedly high-benefit group accounted for $25.2 \%$ and $23.2 \%$ of all patients in the nivolumab and placebo arms, respectively. The $\Delta \Delta$ RMST values of OS and PFS between the markedly low- and high-benefit groups in these triple-factorclassified subgroups were 7.7 and 2.8 months, respectively, which showed the largest difference with statistical significance obtained in this study (Table 4; Fig. 3). No responders were included in the markedly low-benefit group and the objective response rate in the nivolumab arm of the markedly high-benefit group was $21 \%$ (Table 4 ).

\section{Discussion}

The ATTRACTION-2 study demonstrates that nivolumab monotherapy provides benefit to advanced G/GEJ cancer patients who previously received at least two 

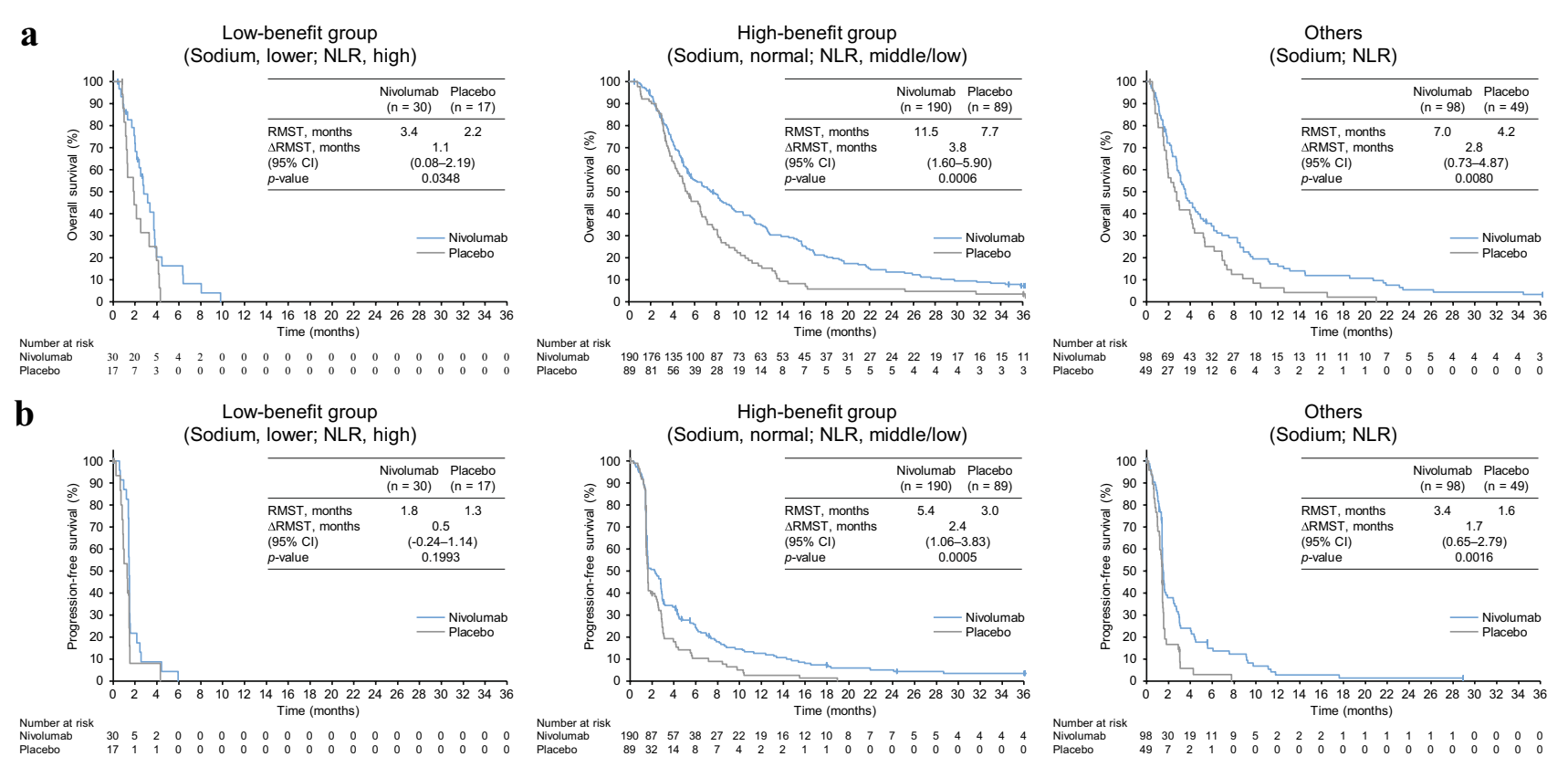
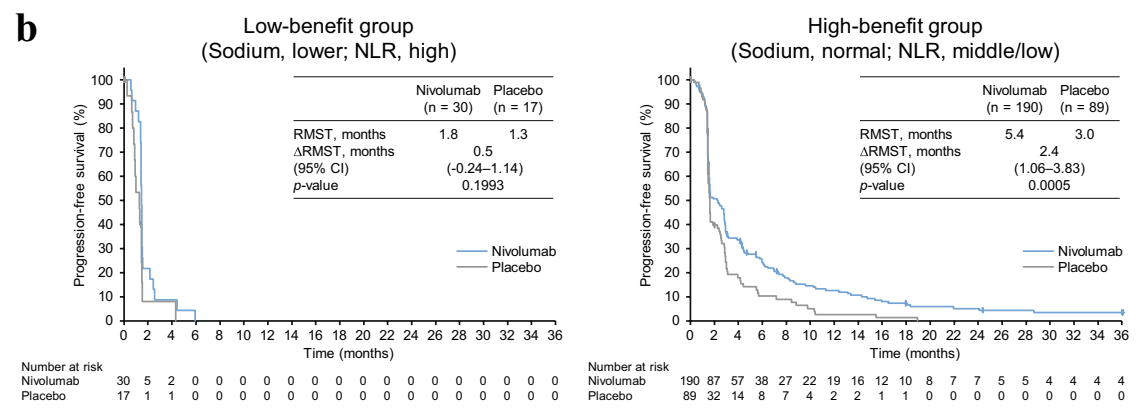

High-benefit group
um, normal; NLR, middle/low)

Fig. 2 Classification by the double-factor combination of serum sodium level and NLR. The Kaplan-Meier curves with the RMST are shown as described in Fig. 1. NLR neutrophil-lymphocyte ratio
Table 3 Double-factor combinations showing statistically significant differences in $\Delta \Delta \mathrm{RMST}$ of both OS and PFS between the lowand high-benefit groups in the exploratory analysis

\begin{tabular}{|c|c|c|c|}
\hline $\begin{array}{l}\text { Factors [classification], low- vs. high- } \\
\text { benefit groups }\end{array}$ & $\begin{array}{l}n \text { (nivolumab/ } \\
\text { placebo) }\end{array}$ & $\begin{array}{l}\text { RMST }^{\mathrm{a}} \text { of OS }(95 \% \\
\mathrm{CI}), \text { months }\end{array}$ & $\begin{array}{l}\Delta \Delta \mathrm{RMST}^{\mathrm{a}} \text { of PFS } \\
(95 \% \mathrm{CI}), \text { months }\end{array}$ \\
\hline Age $[<60]+\mathrm{Na}[$ Lower] vs & $27 / 13$ vs & $6.8(2.57-10.97)$ & $2.8(0.98-4.55)$ \\
\hline Age $[\geq 60]+\mathrm{Na}[$ Normal $]$ & $154 / 77$ & $p=0.0016$ & $p=0.0024$ \\
\hline Age $[<60]+$ P-meta $[$ Yes] vs & $77 / 43$ vs & $6.5(2.43-10.52)$ & $2.8(0.58-5.08)$ \\
\hline Age $[\geq 60]+\mathrm{P}-$ meta $[\mathrm{No}]$ & $93 / 40$ & $p=0.0017$ & $p=0.0138$ \\
\hline $\mathrm{P}$-meta $[$ Yes $]+\mathrm{Na}$ [Lower] vs & $37 / 21$ vs & $6.1(2.52-9.74)$ & $2.1(0.19-3.95)$ \\
\hline P-meta $[\mathrm{No}]+\mathrm{Na}[$ Normal $]$ & $130 / 57$ & $p=0.0009$ & $p=0.0312$ \\
\hline Age $[<60]+$ CL $[$ Lower] vs & $22 / 8 \mathrm{vs}$ & $4.4(1.46-7.41)$ & $2.3(0.61-4.01)$ \\
\hline Age $[\geq 60]+$ CL $[$ Normal $]$ & $162 / 80$ & $p=0.0034$ & $p=0.0077$ \\
\hline CL [Lower] + WBC [Higher] vs & $12 / 7$ vs & $3.5(1.51-5.58)$ & $2.4(1.17-3.71)$ \\
\hline CL $[$ Normal $]+$ WBC $[$ Normal $]$ & $229 / 124$ & $p=0.0006$ & $p=0.0002$ \\
\hline
\end{tabular}

$C I$ confidence interval, $C L$ serum chloride level, $N a$ serum sodium level, $N L R$ neutrophil-lymphocyte ratio, $O S$ overall survival, $P$-meta peritoneal metastasis, $P F S$ progression-free survival, $R M S T$ restricted mean survival time, $W B C$ white blood cell count

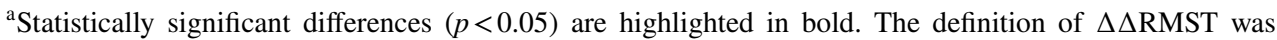
described in "Methods" chemotherapy regimens [13]. In $\mathrm{BaPoFi}$ analysis, we found that combinations of the serum sodium level and either the WBC or NLR effectively classified patients into subgroups associated with low and high benefit from nivolumab. In addition, further exploratory analyses showed that the combination of age, peritoneal metastasis, and serum sodium level defined the markedly low- and high-benefit groups with the largest $\Delta \Delta$ RMST, suggesting that this triple-factor combination would be the most effective predictor of markedly low and high benefit from nivolumab among any combinations tested in this study.
These classifications may be useful because these data are available to clinicians in daily practice.

A combination of the serum sodium level (lower vs. normal range) and $\mathrm{WBC}$ (higher vs. normal range) and that of the serum sodium level and NLR (higher tertile vs. middle/ lower tertile) were associated with significant differences in the $\Delta \Delta$ RMST of both OS and PFS between the low- and high-benefit groups. Low serum sodium levels can occur from various disorders, including dehydration, diarrhea, hypothyroidism, concentrated urine, and heart and kidney failures [20], representing generally worse conditions. Low 
Table 4 Comparison between the markedly low- and highbenefit groups classified by the triple-factor combination of age, peritoneal metastasis, and serum sodium level

\begin{tabular}{|c|c|c|c|c|}
\hline \multirow{3}{*}{$\begin{array}{l}\text { Factors [classification] } \\
\text { Treatment arm }\end{array}$} & \multirow{2}{*}{\multicolumn{2}{|c|}{$\begin{array}{l}\text { Markedly low-benefit group } \\
\text { Age }[<60]+\text { P-meta }[\text { Yes }]+\mathrm{Na} \\
{[\text { Lower] }}\end{array}$}} & \multirow{2}{*}{\multicolumn{2}{|c|}{$\begin{array}{l}\begin{array}{l}\text { Markedly high-benefit } \\
\text { group }\end{array} \\
\text { Age }[\geq 60]+\text { P-meta } \\
{[\mathrm{No}]+\mathrm{Na} \text { [Normal] }}\end{array}$}} \\
\hline & & & & \\
\hline & Nivolumab & Placebo & Nivolumab & Placebo \\
\hline$n$ & 20 & 12 & 80 & 36 \\
\hline$\Delta \Delta \mathrm{RMST}^{\mathrm{a}}$ of OS-months, $(95 \% \mathrm{CI})$ & \multicolumn{4}{|c|}{$7.7(2.20-13.18) ; p=0.0061$} \\
\hline$\Delta \Delta \mathrm{RMST}^{\mathrm{a}}$ of PFS-months, $(95 \% \mathrm{CI})$ & \multicolumn{4}{|c|}{$2.8(0.18-5.50) ; p=0.0366$} \\
\hline$n$ (response assessment population) & 13 & 9 & 75 & 33 \\
\hline \multicolumn{5}{|l|}{ Best overall response- $n(\%)$} \\
\hline Complete response & 0 & 0 & $1(1)$ & 0 \\
\hline Partial response & 0 & 0 & $15(20)$ & 0 \\
\hline Stable disease & $3(23)$ & $1(11)$ & $17(23)$ & $11(33)$ \\
\hline Progressive disease & $6(46)$ & $4(44)$ & $37(49)$ & $21(64)$ \\
\hline Not evaluable & $4(31)$ & $4(44)$ & $5(7)$ & $1(3)$ \\
\hline Objective response rate- $n(\%)$ & 0 & 0 & $16(21)$ & 0 \\
\hline Disease control rate $-n(\%)$ & $3(23)$ & $1(11)$ & $33(44)$ & $11(33)$ \\
\hline
\end{tabular}

$C I$ confidence interval, $N a$ serum sodium level, $O S$ overall survival, $P$-meta peritoneal metastasis, $P F S$ progression-free survival, RMST restricted mean survival time

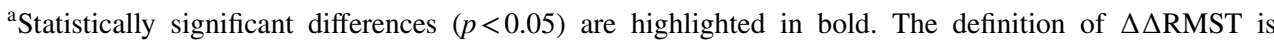
described in "Methods" a
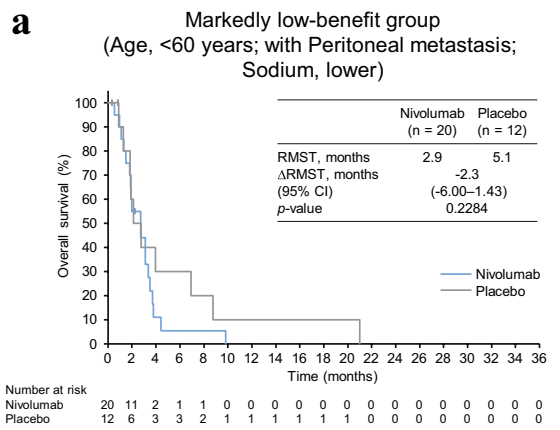

b

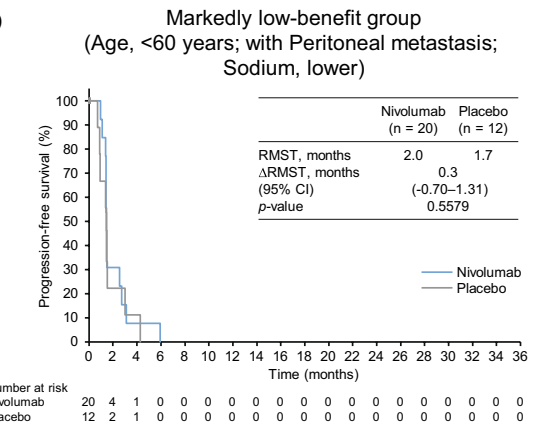

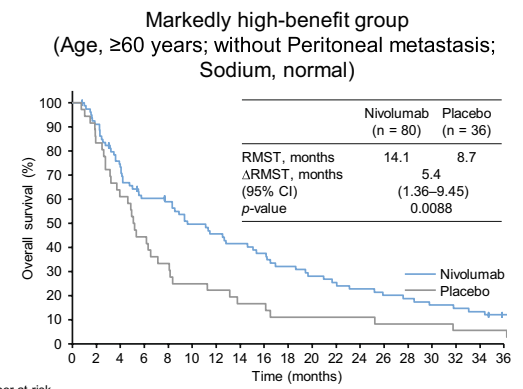

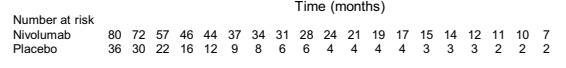

Markedly high-benefit group (Age, $\geq 60$ years; without Peritoneal metastasis;

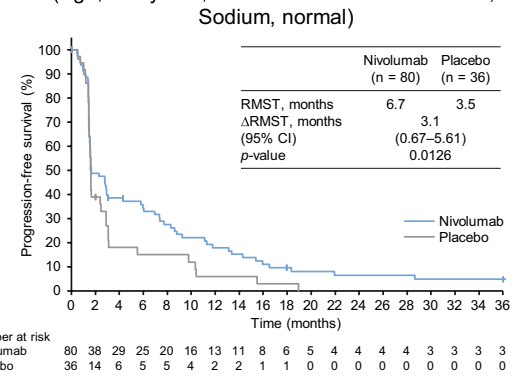

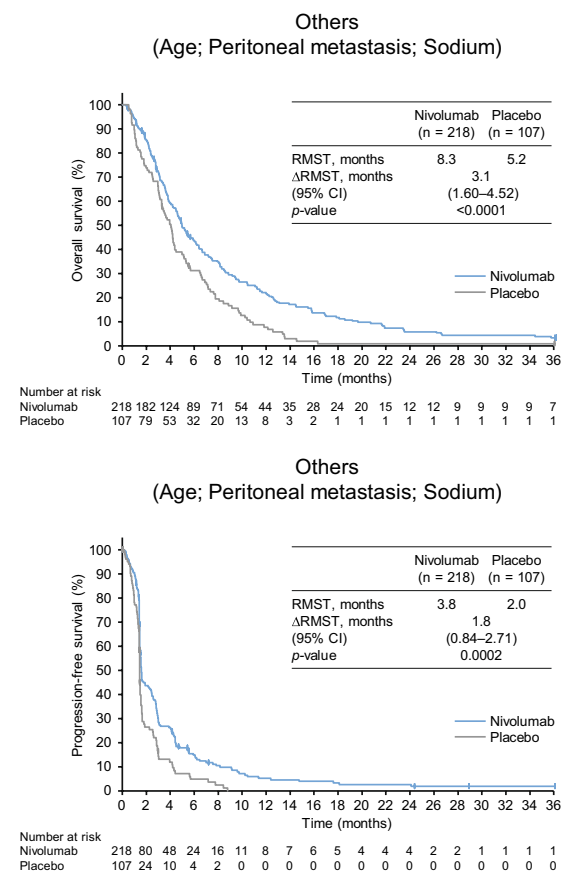

Fig. 3 Classification by the triple-factor combination of age, peritoneal metastasis, and serum sodium level. The Kaplan-Meier curves with the RMST are shown as described in Fig. 1

serum sodium levels have also been reported as a negative prognosis indicator associated with poor PFS for various cancer types [21-23]. In addition, in vitro analyses and in vivo analyses using mouse models have shown that increased serum sodium levels induce interleukin-17-producing helper $\mathrm{T}$ lymphocytes and impair functions of regulatory T lymphocytes [24-26], paradoxically suggesting that a low serum sodium level reduces immune capacity. Indeed, a 
low baseline concentration of sodium in the serum was associated with poor outcomes of ICI therapy for non-small cell lung carcinoma patients [27]. On the other hand, a higher WBC counts and higher NLR would generally account for higher inflammatory states, and a lower lymphocyte level would represent lower immune competency. It has been reported that a higher NLR is an indicator for poor PFS and OS for several cancers, including G/GEJ cancer, treated with ICIs [28-31], and a lower NLR is associated with better response to ICIs [29, 31]. Therefore, it is conceivable that a combination of a low serum sodium level with either a high WBC or high NLR, which represents a low capability of immunosurveillance, would be associated with poor benefit from nivolumab.

Our exploratory analysis showed that the triple-factor combination of an age of 60 years, peritoneal metastasis, and serum sodium levels effectively classified patients into the markedly low- and high-benefit groups and others. Younger patients with gastric cancer at any stage generally had an equivalent or even better OS compared with elderly patients [32-34]. However, when limited to patients with stage IV gastric cancer, some studies showed that OS in younger patients was significantly shorter than that in elderly patients [34]. Some studies also suggested that disease-free survival after surgery of gastric cancer was significantly shorter in younger patients than in elderly patients [32]. Consistently, the RMST value of PFS in patients younger than 60 years was remarkably smaller than that in patients older than 60 years in this study. Considering most patients enrolled in ATTRACTION-2 had recurrent or stage IV gastric cancer, these observations suggest that advanced gastric cancer may be aggressively exacerbated in younger patients, particularly in a salvage treatment setting. Therefore, these patients could receive poor benefit from nivolumab since ICI therapy often induces delayed responses [18, 35]. This study also showed that peritoneal metastasis as a single classification factor was significantly associated with low benefit in OS, but not in PFS, from nivolumab. Consistently, peritoneal metastasis was shown to be associated with poor prognosis in patients with gastric cancer [36, 37], suggesting aggressive tumor progression in these patients. Taking these arguments together, tumors in younger patients with peritoneal metastasis under a generally worse condition including a low immune capacity may rapidly and aggressively progress, resulting in little benefit from nivolumab.

The objective response rate of $21 \%$ in the nivolumab arm of the markedly high benefit group classified by the combination of three factors was comparable to or even higher than those of $15 \%$ and $25 \%$ in patients with $\geq 1$ and $\geq 10$ PD-L1 combined positive score, respectively, who were treated with pembrolizumab monotherapy in the KEYNOTE-062 study [13, 38]. Considering different treatment lines evaluated in ATTRACTION-2 (thirdline or later) and KEYNOTE-062 (first-line), the criteria selecting the markedly high-benefit group may be promising. On the other hand, the disease control rate of $23 \%$ in the nivolumab arm of the markedly low-benefit group was comparable to that $(25 \%)$ in the placebo arm of the whole study population [13], but was higher than that (11\%) in the placebo arm of the markedly low-benefit group. Thus, small but certain benefit from nivolumab might be expected even in the markedly low-benefit group.

Recent researches have been paid attention to biomarkers to enrich the patients expected to have high efficacy of ICIs. However, in ATTRACTION-2, survival benefit of nivolumab was proved in all randomized patients. Therefore, it is not ethical to limit the indication of nivolumab to the enriched patients with high efficacy, excluding patients expected to have small benefits. In clinical practice in this situation, markers to predict patients with low benefit is clinically useful, especially for avoiding early progression.

We acknowledge several limitations in this study. First, this study retrospectively compared patient subgroups classified using multiple factors in an exploratory fashion, raising an issue of multiplicity. Internal validation could not be considered. Any statistically significant differences in this study may cause by chance because no adjustments for multiplicity were performed. In particular, the exploratory analyses assessing any double-factor combinations and the triple-factor combination could be far exploratory. Therefore, it is critical to confirm our findings in a future prospective clinical studies. Second, this study could not consider promising biomarkers including PD-L1 expression, tumor mutation burden, and $\mathrm{CD} 8^{+} \mathrm{T}$ lymphocytes because these data were available only in a limited number of patients in ATTRATION-2. Third, this study focused on nivolumab treatment as salvage therapy. Thus, potential predictors of benefit from nivolumab in earlier treatment lines may be different from those identified in this study. Fourth, we did not consider other cutoff values that would be more appropriate.

In conclusion, this exploratory analysis extracted several double-factor combinations such as the combination of serum sodium levels and either the WBC or NLR as potential predictors of benefit from nivolumab as salvage therapy in advanced G/GEJ cancer patients. In addition, patients younger than 60 years with peritoneal metastasis and with low serum sodium levels may expect markedly low benefit from nivolumab whereas elderly patients without peritoneal metastasis with normal serum sodium level may expect markedly high benefit from nivolumab. Future 
clinical studies with appropriate designs are warranted to confirm these hypotheses.

Supplementary Information The online version contains supplementary material available at https://doi.org/10.1007/s10120-021-01230-4.

Acknowledgements We thank the patients who participated in ATT RACTION-2 and their supportive families. We also thank the investigators and staff at all study sites for their contributions to ATTRAC TION-2. Medical writing support, including drafting of the manuscript, assembling of tables, and creation of images based on the authors' directions, was provided by Masatoshi Esaki, $\mathrm{PhD}$, of Ono Pharmaceutical Co., Ltd., Osaka, Japan, and this study was funded by Ono Pharmaceutical Co., Ltd. and Bristol-Myers Squibb, Princeton, NJ, USA.

Funding This study was funded by Ono Pharmaceutical Co., Ltd., Osaka, Japan, and Bristol-Myers Squibb, Princeton, NJ, USA.

\section{Declarations}

Conflict of interest All authors received grants from Ono Pharmaceutical Co., Ltd., and Bristol-Myers Squibb for the work under consideration for publication. Y-KK reports personal fees from Ono, BristolMyers Squibb, Daehwa, Blueprint, Merck, and Astellas, outside the submitted work. MS reports personal fees from Ono, Bristol-Myers Squibb, AstraZeneca, Chugai, Eli Lilly Japan, MSD, Pfizer Japan, and Taiho, outside the submitted work. TS reports personal fees from Ono for the work under consideration for publication; other from Ono (endorsed Department, lecture fee); grants, personal fees, and other from Chugai and Yakult Honsha (endorsed Department, lecture fee); grants and other from Eli Lilly (lecture fee), grants from MSD, Gilead Sciences, Parexel, and Daiichi Sankyo, and Astellas; grants and personal fees from Taiho, personal fees from Takara-Bio, outside the submitted work. M-HR reports honorarium and advisory board fees from Ono, Bristol-Myers Squibb, MSD, Eli Lilly, Taiho, Novartis, Daiichi Sankyo, AstraZeneca, and Daehwa, outside the submitted work. KK reports research funding from Merck \& Co., Shionogi, Ono, MSD, Merck Serono, and BeiGene, outside the submitted work. HCC reports grants (research support) from Eli Lilly, GlaxoSmithKline, MSD, Merck Serono, Bristol-Myers Squibb, Ono, Taiho, Amgen, BeiGene, Incyte, and Zymework; personal fees (honoraria) from Merck Serono, Eli Lilly, Foundation Medicine, and Roche; and personal fees (consultation) from Taiho, Celltrion, MSD, Eli Lilly, Quintiles, Bristol-Myers Squibb, Merck Serono, Gloria, BeiGene, Amgen, and Zymeworks, outside the submitted work. J-SC reports personal fees (consultation) from Ono for the work under consideration for publication. KMu reports grants and personal fees from Ono, Sanofi, Taiho, and Amgen; grants from MSD, Daiichi Sankyo, Parexel International, Pfizer, Solasia, and Merck Serono; and personal fees from AstraZeneca, Eli Lilly, Chugai, Takeda, Bristol-Myers Squibb, and Bayer, outside the submitted work. K-HY reports personal fees (honoraria) from Ono, Bristol-Myers Squibb, Boehringer Ingelheim, Takeda, MSD, Eli Lilly, Amgen, and Roche, outside the submitted work. TY reports grants and personal fees (honoraria) from Chugai and Taiho; personal fees (honoraria) from Bristol-Myers Squibb; and personal fees (honoraria and advisory role) from Ono, outside the submitted work. TT reports grants from MSD, Merck Serono, and Chugai; and grants and personal fees from Daiichi Sankyo, Takeda, and Taiho, outside the submitted work. K-WL reports grants from MSD, AstraZeneca/MedImmune, Merck KGaA, Pfizer, BeiGene, ALX Oncology, Zymeworks, MacroGenics, Five Prime Therapeutics, Oncologie, Pharmacyclics, Green Cross Corp, ABLBIO, Y-BIOLOGICS, Genexine, LSK BioPharma,
Daiichi Sankyo, Taiho (to his institution for conducting clinical trials), personal fees (consultation) from ISU ABXIS, Bayer and Daiichi Sankyo, outside the submitted work. KC reports personal fees from Ono, Bristol-Myers Squibb, Taiho, and Chugai, outside the submitted work. D-YO reports grants from AstraZeneca outside the submitted work. KMi reports research funding from MSD, Merck Biopharma, Astellas, Taiho., and Daiichi Sankyo, outside the submitted work. HS is an employee of Ono. L-TC reports personal fees from Ono and Bristol-Myers Squibb for the work under consideration for publication; grants from the Ministry of Science and Technology (Taiwan), Ministry of Health and Welfare (Taiwan), grants, personal fees and non-financial support from Novartis (study medication and funding support, and honorarium), grants from Pfizer (study funding), personal fees from Eli Lilly (honorarium), grants from Merck Serono (study funding), grants, personal fees and non-financial support from TTY (study medication and funding support, and honorarium), personal fees from PharmaEngine, Shire, MSD, Bristol-Myers Squibb, and Ono (honorarium), grants from OBI (preclinical testing), grants from Polaris (translational Research funding), grants, personal fees and nonfinancial support from SynCore (study medication and funding support, and honorarium), grants and non-financial support from Celgene (study medication and funding), and personal fees from Five Prime, and Merrimack' outside the submitted work. NB reports personal fees from Ono and Bristol-Myers Squibb for the work under consideration for publication; grants and personal fees from Taiho and grants from Takeda, outside the submitted work.

Ethical standards All procedures followed the ethical standards of the responsible committee on human experimentation (institutional and national) and the Helsinki Declaration of 1964 and later versions.

Informed consent Informed consent to be included in the ATTRAC TION-2 study was obtained from all patients.

Open Access This article is licensed under a Creative Commons Attribution 4.0 International License, which permits use, sharing, adaptation, distribution and reproduction in any medium or format, as long as you give appropriate credit to the original author(s) and the source, provide a link to the Creative Commons licence, and indicate if changes were made. The images or other third party material in this article are included in the article's Creative Commons licence, unless indicated otherwise in a credit line to the material. If material is not included in the article's Creative Commons licence and your intended use is not permitted by statutory regulation or exceeds the permitted use, you will need to obtain permission directly from the copyright holder. To view a copy of this licence, visit http://creativecommons.org/licenses/by/4.0/.

\section{References}

1. Chen DS, Mellman I. Oncology meets immunology: the cancerimmunity cycle. Immunity. 2013;39:1-10.

2. Xu C, Chen YP, Du XJ, Liu JQ, Huang CL, Chen L, et al. Comparative safety of immune checkpoint inhibitors in cancer: systematic review and network meta-analysis. BMJ. 2018;363:k4226.

3. Darvin P, Toor SM, Sasidharan Nair V, Elkord E. Immune checkpoint inhibitors: recent progress and potential biomarkers. Exp Mol Med. 2018;50:165.

4. Marrone KA, Ying W, Naidoo J. Immune-related adverse events from immune checkpoint inhibitors. Clin Pharmacol Ther. 2016;100:242-51. 
5. Buder-Bakhaya K, Hassel JC. Biomarkers for clinical benefit of immune checkpoint inhibitor treatment-a review from the melanoma perspective and beyond. Front Immunol. 2018;9:1474.

6. Gibney GT, Weiner LM, Atkins MB. Predictive biomarkers for checkpoint inhibitor-based immunotherapy. Lancet Oncol. 2016;17:e542-51.

7. Davis AA, Patel VG. The role of PD-L1 expression as a predictive biomarker: an analysis of all US Food and Drug Administration (FDA) approvals of immune checkpoint inhibitors. J Immunother Cancer. 2019;7:278.

8. Brahmer JR, Drake CG, Wollner I, Powderly JD, Picus J, Sharfman WH, et al. Phase I study of single-agent anti-programmed death-1 (MDX-1106) in refractory solid tumors: safety, clinical activity, pharmacodynamics, and immunologic correlates. J Clin Oncol. 2010;28:3167-75.

9. Yang Q, Xu Z, Zheng L, Zhang L, You Q, Sun J. Multimodal detection of PD-L1: reasonable biomarkers for immune checkpoint inhibitor. Am J Cancer Res. 2018;8:1689-96.

10. Marabelle A, Fakih M, Lopez J, Shah M, Shapira-Frommer R, Nakagawa K, et al. Association of tumour mutational burden with outcomes in patients with advanced solid tumours treated with pembrolizumab: prospective biomarker analysis of the multicohort, open-label, phase 2 KEYNOTE-158 study. Lancet Oncol. 2020;21:1353-65.

11. Marcus L, Lemery SJ, Keegan P, Pazdur R. FDA approval summary: pembrolizumab for the treatment of microsatellite instability-high solid tumors. Clin Cancer Res. 2019;25:3753-8.

12. Darabi S, Braxton DR, Eisenberg BL, Demeure MJ. Predictive biomarkers for immunotherapy response beyond PD-1/PD-L1. Oncology (Williston Park). 2020;34:321-7.

13. Kang Y-K, Boku N, Satoh T, Ryu M-H, Chao Y, Kato K, et al. Nivolumab in patients with advanced gastric or gastro-oesophageal junction cancer refractory to, or intolerant of, at least two previous chemotherapy regimens (ONO-4538-12, ATTRAC TION-2): a randomised, double-blind, placebo-controlled, phase 3 trial. Lancet. 2017;390:2461-71.

14. Boku N, Satoh T, Ryu M-H, Chao Y, Kato K, Chung HC, et al. Nivolumab in previously treated advanced gastric cancer (ATT RACTION-2): 3-year update and outcome of treatment beyond progression with nivolumab. Gastric Cancer. in press.

15. Nugent C, Guo W, Müller P, Ji Y. Bayesian approaches to subgroup analysis and related adaptive clinical trial designs. JCO Precis Oncol. 2019;1-9.

16. Morita S, Müller P. Bayesian population finding with biomarkers in a randomized clinical trial. Biometrics. 2017;73:1355-65.

17. Uno H, Claggett $B$, Tian L, Inoue E, Gallo P, Miyata $T$, et al. Moving beyond the hazard ratio in quantifying the between-group difference in survival analysis. J Clin Oncol. 2014;32:2380-5.

18. Liang F, Zhang S, Wang Q, Li W. Treatment effects measured by restricted mean survival time in trials of immune checkpoint inhibitors for cancer. Ann Oncol. 2018;29:1320-4.

19. A'Hern RP. Restricted mean survival time: an obligatory end point for time-to-event analysis in cancer trials? J Clin Oncol. 2016;34:3474-6.

20. Lee JJY, Kilonzo K, Nistico A, Yeates K. Management of hyponatremia. Can Med Assoc J. 2014;186:E281-6.

21. Jeppesen AN, Jensen HK, Donskov F, Marcussen N, Von Der Maase $\mathrm{H}$. Hyponatremia as a prognostic and predictive factor in metastatic renal cell carcinoma. Br J Cancer. 2010;102:867-72.

22. Poonyam P, Aumpan N, Vilaichone RK. Prognostic factors for survival in patients with gastric adenocarcinoma. Cancer Rep. 2020; 1305 .
23. Castillo JJ, Glezerman IG, Boklage SH, Chiodo J, Tidwell BA, Lamerato LE, et al. The occurrence of hyponatremia and its importance as a prognostic factor in a cross-section of cancer patients. BMC Cancer. 2016;16:564.

24. Hernandez AL, Kitz A, Wu C, Lowther DE, Rodriguez DM, Vudattu N, et al. Sodium chloride inhibits the suppressive function of FOXP3+ regulatory T cells. J Clin Invest. 2015;125:4212-22.

25. Kleinewietfeld M, Manzel A, Titze J, Kvakan H, Yosef N, Linker RA, et al. Sodium chloride drives autoimmune disease by the induction of pathogenic TH 17 cells. Nature. 2013;496:518-22.

26. Wu C, Yosef N, Thalhamer T, Zhu C, Xiao S, Kishi Y, et al. Induction of pathogenic TH 17 cells by inducible salt-sensing kinase SGK1. Nature. 2013;496:513-7.

27. Fucà G, Galli G, Poggi M, Lo Russo G, Proto C, Imbimbo M, et al. Low baseline serum sodium concentration is associated with poor clinical outcomes in metastatic non-small cell lung cancer patients treated with immunotherapy. Target Oncol. 2018;13:795-800.

28. Xie X, Liu J, Yang H, Chen H, Zhou S, Lin H, et al. Prognostic value of baseline neutrophil-to-lymphocyte ratio in outcome of immune checkpoint inhibitors. Cancer Invest. 2019;37:265-74.

29. Formica V, Morelli C, Patrikidou A, Murias C, Butt S, Nardecchia A, et al. Gastric inflammatory prognostic index (GIPI) in patients with metastatic gastro-esophageal junction/gastric cancer treated with PD-1/PD-L1 immune checkpoint inhibitors. Target Oncol. 2020;15:327-36.

30. Sacdalan DB, Lucero JA, Sacdalan DL. Prognostic utility of baseline neutrophil-to-lymphocyte ratio in patients receiving immune checkpoint inhibitors: a review and meta-analysis. Onco Targets Ther. 2018;11:955-65.

31. Kargl J, Zhu X, Zhang H, Yang GHY, Friesen TJ, Shipley M, et al. Neutrophil content predicts lymphocyte depletion and anti-PD1 treatment failure in NSCLC. JCI Insight. 2019;4.

32. Ramos MFKP, Pereira MA, Sagae VMT, Mester M, Morrell ALG, Dias AR, et al. Gastric cancer in young adults: a worse prognosis group? Rev Col Bras Cir. 2019;46:20192256.

33. Eguchi T, Takahashi Y, Yamagata M, Kasahara M, Fujii M. Gastric cancer in young patients. J Am Coll Surg. 1999;188:22-6.

34. Tekesin K, Emin Gunes M, Tural D, Akar E, Zirtiloglu A, Karaca $\mathrm{M}$, et al. Clinicopathological characteristics, prognosis and survival outcome of gastric cancer in young patients: a large cohort retrospective study. J BUON. 2019;24:672-8.

35. Robert C. A decade of immune-checkpoint inhibitors in cancer therapy. Nat Commun. 2020;11:10-2.

36. Wang Z, Chen J-Q, Liu J-L, Tian L. Issues on peritoneal metastasis of gastric cancer: an update. World J Surg Oncol. 2019;17:215.

37. Kanda M, Kodera Y. Molecular mechanisms of peritoneal dissemination in gastric cancer. World J Gastroenterol. 2016;22:6829-40.

38. Shitara K, Van Cutsem E, Bang YJ, Fuchs C, Wyrwicz L, Lee $\mathrm{KW}$, et al. Efficacy and safety of pembrolizumab or pembrolizumab plus chemotherapy vs chemotherapy alone for patients with first-line, advanced gastric cancer: the KEYNOTE-062 phase 3 randomized clinical trial. JAMA Oncol. 2020;6:1571-80.

Publisher's Note Springer Nature remains neutral with regard to jurisdictional claims in published maps and institutional affiliations. 


\section{Authors and Affiliations}

\section{Yoon-Koo Kang ${ }^{1}$ (1) - Satoshi Morita ${ }^{2} \cdot$ Taroh Satoh $^{3} \cdot$ Min-Hee Ryu $^{1} \cdot$ Yee Chao ${ }^{4} \cdot K_{\text {Ken Kato }}{ }^{5}$ Hyun Cheol Chung ${ }^{6}$. Jen-Shi Chen ${ }^{7}$. Kei Muro ${ }^{8}$. Won Ki Kang ${ }^{9} \cdot$ Kun-Huei Yeh ${ }^{10}$. Takaki Yoshikawa ${ }^{11,26}$. Sang Cheul Oh ${ }^{12} \cdot$ Li-Yuan Bai $^{13}$. Takao Tamura ${ }^{14,27}$. Keun-Wook Lee ${ }^{15}$. Yasuo Hamamoto ${ }^{16}$. Jong Gwang Kim ${ }^{17} \cdot K_{\text {Keisho Chin }}{ }^{18}$. Do-Youn Oh ${ }^{19}$. Keiko Minashi ${ }^{20} \cdot$ Jae Yong Cho ${ }^{21} \cdot$ Masahiro Tsuda $^{22} \cdot$ Hiroki Sameshima $^{23} \cdot$ Li-Tzong Chen $^{24,25} \cdot$ Narikazu Boku $^{5}$}

1 Department of Oncology, Asan Medical Center, University of Ulsan College of Medicine, Seoul, South Korea

2 Department of Biomedical Statistics and Bioinformatics, Kyoto University Graduate School of Medicine, Kyoto, Japan

3 Frontier Science for Cancer and Chemotherapy, Osaka University Graduate School of Medicine, Suita, Japan

4 Department of Oncology, Taipei Veterans General Hospital, Taipei, Taiwan

5 Division of Gastrointestinal Medical Oncology, National Cancer Center Hospital, Tokyo, Japan

6 Department of Internal Medicine, Yonsei Cancer Center, Yonsei University College of Medicine, Seoul, South Korea

7 Division of Hematology and Oncology, Department of Internal Medicine, Linkou Chang Gung Memorial Hospital, Chang Gung University, Taoyuan, Taiwan

8 Department of Clinical Oncology, Aichi Cancer Center Hospital, Nagoya, Japan

9 Division of Hematology-Oncology, Department of Medicine, Samsung Medical Center, Sungkyunkwan University School of Medicine, Seoul, South Korea

10 Department of Oncology, National Taiwan University Hospital, Taipei, Taiwan

11 Department of Gastrointestinal Surgery, Kanagawa Cancer Center, Yokohama, Japan

12 Division of Hematology and Oncology, Department of Internal Medicine, College of Medicine, Korea University, Seoul, South Korea

13 Division of Hematology and Oncology, Department of Internal Medicine, China Medical University Hospital, China Medical University, Taichung, Taiwan

14 Department of Medical Oncology, Faculty of Medicine, Kindai University, Osakasayama, Japan
15 Division of Hematology and Oncology, Department of Internal Medicine, Seoul National University Bundang Hospital, Seoul National University College of Medicine, Seongnam, South Korea

16 Keio Cancer Center, Keio University School of Medicine, Tokyo, Japan

17 Kyungpook National University School of Medicine, Daegu, South Korea

18 Department of Gastroenterology, Cancer Institute Hospital of the Japanese Foundation for Cancer Research, Tokyo, Japan

19 Seoul National University Hospital, Cancer Research Institute, Seoul National University College of Medicine, Integrated Major in Innovative Medical Science, Seoul National University Graduate School, Seoul, South Korea

20 Department of Clinical Trial Promotion, Chiba Cancer Center, Chiba, Japan

21 Department of Medical Oncology, Gangnam Severance Hospital, Yonsei University College of Medicine, Seoul, South Korea

22 Department of Gastroenterological Oncology, Hyogo Cancer Center, Akashi, Japan

23 Medical Affairs, Ono Pharmaceutical, Co. Ltd., Osaka, Japan

24 National Institute of Cancer Research, National Health Research Institutes, Tainan, Taiwan

25 Kaohsiung Medical University Hospital, Kaohsiung Medical University, Kaohsiung, Taiwan

26 Present Address: Department of Gastric Surgery, National Cancer Center Hospital, Tokyo, Japan

27 Present Address: Department of Medical Oncology, Kindai University Nara Hospital, Ikoma, Japan 